\title{
Interação do exercício de natação sustentada e da densidade de estocagem no desempenho e na composição corporal de juvenis de matrinxã Brycon amazonicus
}

\author{
Sustained swimming and stocking density interaction in the performance and body composition of \\ matrinxã Brycon amazonicus juveniles
}

\author{
Gustavo Alberto Arbeláez-Rojas ${ }^{\mathrm{I}}$ Gilberto Moraes $^{{ }^{*}}$
}

RESUMO

O objetivo deste estudo foi estimar o efeito da densidade de estocagem associada ao exercício de natação moderada no desempenho e na composição corporal de juvenis de matrinxã (Brycon amazonicus). Foram utilizados peixes com comprimento e peso médio inicial de $12,3 \pm 0,5 \mathrm{~cm}$ e $18,4 \pm 0,1 \mathrm{~g}$, os quais foram distribuídos ao acaso em dois grupos: o primeiro grupo de peixes foi estocado em três densidades correspondendo a 88, 176 e 353 peixes $m^{-3}$ e foi condicionado a nadar a uma velocidade de $1,0 c c \mathrm{~s}^{-1}$ (comprimento corporal por segundo) em tanques circulares de 250L, durante 70 dias. $O$ segundo grupo de peixes foi mantido nas mesmas densidades em água parada (sem exercício) perfazendo no total seis tratamentos com três repetições. Foram estimados parâmetros de desempenho e da composição corporal, particularmente do músculo branco e do músculo vermelho de ambos os grupos. Os resultados mostraram que o exercício e a densidade afetaram significativamente o crescimento e a composição dos músculos do matrinxã. O grupo de peixes criados sob exercício moderado na densidade de 176 peixes $\mathrm{m}^{-3}$ apresentou melhor desempenho $(P<0,05)$, que se refletiu em maior crescimento e uniformidade do peso, além de melhor fator de condição, conversão alimentar e sobrevivência. Além disso, o exercício de natação sustentada aumentou tanto a concentração de proteína no músculo branco, como a deposição de lipídios no músculo vermelho nesse mesmo grupo de peixes. Já em altas densidades de estocagem, os resultados foram contrários. Portanto, a criação de juvenis de matrinxã sob condições de exercício moderado e em densidades próximas a 176 peixes $m^{-3}$ é a mais indicada, já que se foram observadas as melhores respostas de crescimento e desempenho, além de mudanças benéficas na composição corporal. Essas mudanças resultaram em melhor condicionamento físico, possibilitando, assim, maior tolerância a altas densidades de criação.

Palavras-chave: Brycon amazonicus, recria de peixes, cultivo intensivo, raceway, gordura, matrinxã.

\begin{abstract}
The aim of the present study was to gauge the effect of stocking density associated to the sustained swimming on the performance and body composition of matrinxã (Brycon amazonicus) juveniles. The fish were initially sized at $12.3 \pm 0.5 \mathrm{~cm}$ length and $18.4 \mathrm{~g} \pm 0.1 \mathrm{~g}$ weight. They were distributed randomly in two groups: the first was arranged into three densities of 88, 176 and 353 fish $\mathrm{m}^{-3}$ and conditioned to swim at 1.0BL sec-1 in circular tanks of $250 \mathrm{~L}$ for 70 days; the second was arranged in the same fish densities but in static waters performing six treatments with three repetitions. Performance and body compositions were estimated in white and red muscles for both groups. The results express the growth and muscle composition change in response to the exercise and fish density. The fish maintained in moderate swimming at 176 fish $\mathrm{m}^{-3}$ depicted the best performance $(P<0.05)$, reflected as best growth, weight uniformity, conditioning factor, feed conversion and survival. Otherwise, the sustained swimming enhanced the white muscle protein concentration and the red muscle lipid deposition in the same group of fish. Yet, the results were opposite in high fish densities. Therefore, the rearing of matrinxã juveniles under moderate sustained swimming and near to 176 fish $\mathrm{m}^{-3}$ is suggested, since the best responses of growth and performance, and beneficial body changes were observed. These responses resulted in better body conditioning allowing higher tolerance to high fish densities.
\end{abstract}

Key words: Brycon amazonicus, fish farming, intensive rearing, raceway, fat, matrinxã.

\section{INTRODUÇÃO}

Densidades elevadas são utilizadas em sistemas intensivos de produção de peixes, visando

'Departamento de Genética e Evolução, Universidade Federal de São Carlos (UFSCAR), Rod. Washington Luis, km 235, 13565905, São Carlos, SP, Brasil. E-mail: gil@power.ufscar.br. *Autor para correspondência. 
maximizar tanto o uso da água, quanto do espaço em prol de maior produtividade e rentabilidade (PAPST et al., 1992). No entanto, a criação de peixes em pequeno espaço pode gerar situações de estresse crônico que venham desequilibrar a homeostase (BARTON, 2002) e incidir em alterações bioquímicas, fisiológicas e comportamentais (ARANÄRÄ \& BRÄNNÄS, 1996; WEDEMEYER, 1997). Pode ainda levar a mudanças significativas no crescimento e no desempenho. Isso se deve à severa demanda metabólica, chegando inclusive a mobilizar importantes reservas energéticas, como os lipídios, e modificar a composição corporal dos peixes (VIJAYAN et al., 1990), com subseqüente perda de peso. Todas essas alterações podem levar a uma redução da condição física, da eficiência alimentar e ao aumento da heterogeneidade no tamanho (ELLIS et al., 2002).

Estes efeitos podem ser resultantes de práticas inapropriadas de manejo alimentar (suprimento restrito em termos de quantidade no espaço ou no tempo) e acabam gerando a competição pelo alimento e exaltando agressividade nos peixes (KESTEMONT \& BARAS, 2001). Entretanto, quando a densidade de estocagem (DE) é ajustada e otimizada, o comportamento agressivo pode ser modificado ou ainda reduzido quando os peixes são expostos a correntes moderadas da água. Nessas condições, ao invés de investir boa parte da energia adquirida em defesa do território, os peixes a canalizam para o crescimento (JOBLING et al., 1993). A corrente da água estimularia os peixes a nadar ativamente e a formar cardumes (locomoção sincronizada). Com isso, praticamente desaparece a territorialidade e melhora o ambiente social, permitindo um consumo mais homogêneo do alimento, o qual se pode refletir em taxas de crescimento mais uniformes (JOBLING, 1994).

O matrinxã, Brycon amazonicus (SPIX \& AGASSIZ, 1829), é uma espécie nativa das bacias Amazônica e Tocantins-Araguaia (HOWES, 1982) de grande potencial para a aqüicultura por apresentar alta taxa de crescimento, ótimo desempenho em cativeiro e alta qualidade e sabor de sua carne (ROCHA et al., 1982; ZANIBONI FILHO et al., 2006). Estudos demonstram que a espécie é bem tolerante a procedimentos de captura e ao adensamento sem mostrar sinais de estresse (ROCHA et al. 2004; BRANDÃO et al., 2005). Além disso, as suas características reofílicas, a capacidade de nadar longas distâncias a fim de se reproduzir e formato hidrodinâmico de seu corpo permitem boas respostas a testes de crescimento tanto no campo, quanto no laboratório sob condições de exercício moderado (ARBELÁEZ-ROJAS et al., 2007; HACKBARTH \&
MORAES, 2006). Desse modo, é importante avaliar o potencial de crescimento do matrinxã, já que exibe um modelo biológico que pode ser explorado de forma mais eficiente por meio do exercício moderado como estratégia alternativa de manejo para maximizar seu crescimento em sistemas intensivos. Sendo assim, o presente estudo teve como objetivo avaliar o efeito do exercício de natação moderada associado à densidade de estocagem no desempenho e na composição corporal de juvenis de matrinxã.

\section{MATERIAL E MÉTODOS}

Juvenis de matrinxã foram adquiridos em piscicultura comercial do interior do Estado de São Paulo. Os peixes foram transportados até as instalações experimentais do Laboratório de Bioquímica Adaptativa, da Universidade Federal de São Carlos, para um período de quarentena. Os parâmetros da qualidade da água monitorados diariamente foram: concentração de oxigênio dissolvido e temperatura. Semanalmente era verificada a amônia total- $\mathrm{N}=\left(\mathrm{NH}_{3}+\mathrm{NH}_{4}^{+}\right), \mathrm{pH}$ e condutividade elétrica. Em seguida, foram alojados em tanques circulares de 2000L, acoplados a um sistema fechado de recirculação de água termostatizada, aerada e filtrada. Após um período de adaptação de três semanas, quando atingiram um comprimento e o peso médio de $12,3 \pm 0,5 \mathrm{~cm}$ e $18,4 \pm 0,1 \mathrm{~g}$, respectivamente, foram aleatoriamente distribuídos em dois grupos. Um grupo foi forçado a nadar a $1 \mathrm{cc} / \mathrm{s}$ (comprimento corporal por segundo) e arranjado em três densidades de estocagem; 15,30 e 60 peixes por tanque (volume útil 170 litros de água cada), o que correspondeu a 88,176 e 353 peixes $\mathrm{m}^{-3}$. O outro grupo foi também submetido às mesmas taxas de estocagem, mas mantidos em condições de água parada (sem exercício) e considerado como grupo controle ou de referência. Os peixes eram alimentados próximo à saciedade aparente três vezes ao dia, com ração comercial extrusada Purina contendo $32 \%$ de proteína bruta (PB). Durante um período de 70 dias, os peixes foram submetidos às condições experimentais, e quatro biometrias foram realizadas: três ao final de cada 21 dias e a última aos 70 dias. As amostragens abrangiam todos os peixes de cada tanque. Foi adotado um delineamento inteiramente casualizado, com seis tratamentos e três repetições em esquema fatorial de $3 \times 2$, constituídos por três densidades de estocagem e duas condições físicas, com ou sem exercício.

Foram selecionados tanques circulares com o fundo em forma de cone invertido com $20^{\circ} \mathrm{de}$ inclinação (tanques autolimpantes) a fim de facilitar a remoção da água e da matéria orgânica. Foi colocado na parte central do fundo de cada tanque um sistema

Ciência Rural, v.39, n.1, jan-fev, 2009. 
de canos de PVC de duas polegadas de diâmetro para permitir o escoamento e direcionamento da água de cada tanque para uma caneleta de condução para tratamento, constituindo-se assim um sistema fechado com recirculação. A correnteza em cada tanque foi criada pela passagem forçada da água através de um sistema em "L" de canos em PVC de $1 / 4$ de polegada, com as hastes posicionadas horizontal e verticalmente. A força e direção do jato de água foram ajustadas para regular sua velocidade através do fluxo gerado por uma bomba marca "Mark", modelo NXDP4, com entrada e saída de uma polegada e $1 \mathrm{CV}$ de potência. A velocidade da água em cada tanque era aferida e ajustada a cada três semanas com um fluxômetro mecânico (General Oceanics. Inc. Miami. Florida). A velocidade de natação selecionada foi de $1 \mathrm{cc} \mathrm{s}^{-1}$, baseada em estudos anteriores (ARBELÁEZ-ROJAS, 2007), que apontaram essa velocidade como benéfica para o crescimento de matrinxã. O grupo controle foi mantido sedentário em tanques com entrada de água por cano posicionado horizontalmente sobre a caixa para não criar correnteza. Mediante um sistema de recirculação, os parâmetros da qualidade da água dos tanques foram mantidos dentro da faixa dos tanques iniciais de 2000L. Para isso, a água passava através de um sistema servido por filtragem mecânica e biológica e aerada através de um sistema de difusores alimentados com soprador. A temperatura era mantida com sistema de aquecimento individual automatizado, enquanto que o $\mathrm{pH}$ da água foi estabilizado com conchas marinas, posicionadas na saída do filtro mecânico.

Os peixes eram anestesiados com óleo de cravo previamente diluído em etanol e ajustado para uma concentração final de $40 \mathrm{mg} \mathrm{L}^{-1}$ (INOUE et al. 2003), medidos em ictiômetro graduado em $\mathrm{mm}$, e pesados em balança digital de $0,1 \mathrm{~g}$. Foram calculados o crescimento em peso e em comprimento, a taxa de crescimento

específico TCE $=\left[\frac{\left(\ln P_{f}-\ln P_{i}\right)}{\Delta t}\right] \times 100$; o fator de

condição $\mathrm{K}=\left(\mathrm{w}_{\mathrm{t}} / \mathrm{L}_{\mathrm{t}}\right)^{\mathrm{b}}$, (em que: $\mathrm{W}_{\mathrm{t}}=$ peso total; $\mathrm{L}_{\mathrm{t}}=$ comprimento total e b: coeficiente angular da regressão entre $\mathrm{w}_{\mathrm{t}} / \mathrm{L}_{\mathrm{t}}$ ); a conversão alimentar $\mathrm{CA}=$ consumo de ração/ganho de peso; e o índice de uniformidade em peso de cada tratamento. Esse índice foi obtido pela razão entre o número de animais com peso compreendido entre $20 \%$ do peso médio e o número total de animais multiplicado por 100, de acordo com a expressão $U=\frac{N_{ \pm 20 \%}}{N_{t}} \times 100$ (FURUYA et al. 1998).

Após a biometria os peixes retornavam às condições originais. Ao final dos 70 dias de experimento, seis exemplares de cada tanque foram amostrados para análise da composição corporal com base em amostras coletadas de músculo branco e músculo vermelho. Foram determinadas proteína total, extrato etéreo e umidade (AOAC 1990).

Os dados foram submetidos à análise de variância de dois fatores, e as médias foram comparadas pelo teste de Tukey a 5\% de probabilidade. Os dados de sobrevivência e uniformidade do lote passaram por transformação angular antes da análise estatística, a qual foi executada pelo programa Statistical Analyses System (SAS), version 8.0. O presente trabalho foi previamente avaliado pela Comissão de Ética para procedimentos com experimentação animal da UFSCar, tendo sido aprovado e protocolado sob o número 042107.

\section{RESULTADOS E DISCUSSÃO}

Os parâmetros da qualidade da água mantiveram-se inalterados. No entanto, no último terço do período experimental, foi dada especial atenção à água do grupo de peixes estocados em alta densidade, em que a concentração e saturação do oxigênio dissolvido (OD) tenderam a diminuir para níveis mínimos de 3,0mg L-1 ou 60\%. Quando a concentração de OD tende a cair abaixo desses patamares, o crescimento e a saúde dos peixes começam a se deteriorar (BOYD, 1990; VINATEA, 2004). Não obstante, durante o experimento o valor médio de OD foi de $4,7 \pm 0,8 \mathrm{mg} \mathrm{l}^{-1}$, equivalente a $71 \%$ de saturação. A temperatura da água do sistema de recirculação manteve-se em $28,1 \pm 0,9^{\circ} \mathrm{C}$. $\mathrm{O}$ valor de $\mathrm{pH}$ foi 7,2 $\pm 0,5$, estando dentro da faixa de conforto para a criação de peixes (BOYD 1990). Adureza foi de $88 \pm 5,0 \mu \mathrm{S} \mathrm{cm}^{-1}$ e as concentrações de amônia não-ionizada (NH ) estiveram abaixo de $0,03 \mathrm{mg} \mathrm{L}^{-1}$ durante o período experimental.

Os resultados de desempenho obtidos pelos juvenis de matrinxã (Tabela 1), mostram que o crescimento variou significativamente em função tanto da densidade de estocagem, quanto da condição física. O grupo de peixes criados na densidade intermediária (DI) e sob exercício contínuo-moderado atingiu peso final $(\mathrm{P}<0,05)$ maior que os demais grupos. Destaca-se que o crescimento desse grupo foi $34 \%$ maior $(\mathrm{P}<0,05)$, quando comparado ao grupo de peixes criados na mesma densidade, porém, sem exercício. Além disso, foi observado que o exercício moderado teve um efeito positivo no desempenho dos peixes alojados nas diferentes densidades, já que o crescimento deles foi homogêneo, sem diferenças no peso final. Principalmente, o peso global de todos eles foi, em média, $27 \%$ maior $(\mathrm{P}<0,05)$ que o peso médio dos peixes 
criados nas mesmas densidades e que não realizaram exercício induzido.

$\mathrm{O}$ índice de uniformidade em peso é um importante indicador para a piscicultura (FURUYA et al., 1998). No presente estudo, os peixes que foram exercitados e estocados na densidade intermediária apresentaram melhor uniformidade do peso que os demais grupos de peixes. $\mathrm{Na}$ avaliação da densidade de estocagem, foi verificado aumento desse índice concomitante à densidade $(\mathrm{P}<0,05)$. Estes dados são condizentes com os relatos de MARQUES et al (2004). No entanto, GOMES et al. (2000) encontraram aumento na variação do tamanho de larvas de matrinxã em resposta ao aumento da densidade. Em outro estudo em taque-rede com matrinxã, não foram constatadas diferenças significativas no coeficiente de variação de crescimento (BRANDÃO et al., 2005).

Em um estudo em que foram avaliados ambos os fatores, densidade e exercício, sobre a performance do Artic charr (Salvelinus alpinus), CHRISTIANSEN et al. (1992) constataram que essa espécie cresce melhor quando exercitada e criada em baixas densidades (biomassa inicial $23 \mathrm{~kg} \mathrm{~m}^{-3}$ ) do que quando criada em água parada. Não obstante, em altas densidades (biomassa inicial $47 \mathrm{~kg} \mathrm{~m}^{-3}$ ), as respostas de crescimento em ambas as condições foram iguais entre si e similares aos peixes mantidos em baixa densidade.

Apesar de existir um consenso geral de que densidades demasiado elevadas têm efeito prejudicial sobre o crescimento dos peixes, esses efeitos dependem do comportamento da espécie e da sua acomodação às condições de ambientes de adensamento em espaços reduzidos (ELLIS et al., 2002). De fato, o matrinxã consegue se adaptar e apresentar bom desempenho de crescimento, sem mostrar sinais de estresse, quando confinado em altas densidades populacionais. MARQUES et al. (2004), avaliando o crescimento de matrinxã em densidades de 24, 48, 72 e 96 peixes $\mathrm{m}^{-3}$, observaram melhores resultados com 96 peixes $\mathrm{m}^{-3}$. Em outro estudo, em que foi quadruplicada a densidade de estocagem de 0,5 para 2 peixes $\mathrm{m}^{-3}$, não foram observados sinais de estresse (ROCHA et al, 2004). O mesmo resultado é relatado por BRANDÃO et al. (2005), os quais estocaram alevinos de matrinxã em tanque rede, em densidades de 200, 300, 400 e 500 peixes $\mathrm{m}^{-3}$. Ao final de 60 dias de criação, o melhor desempenho atingido foi observado na densidade máxima.

Diversos estudos indicam que o exercício sustentado promove e acelera o crescimento de forma significativa em salmonídeos (DAVISON \& GOLDSPINK, 1977; TOTLAND et al., 1987; JOBLING et al., 1993; DAVISON, 1997), no bagre do canal (JARBOE \& GRANT, 1996), no matrinxã (HARBARTH \& MORAES, 2006), promovendo mudanças marcantes na fisiologia, bioquímica e morfologia dos músculos propulsores dos peixes. É preciso considerar ainda o fato de que esses músculos representam entre 50 e $60 \%$ do peso vivo (JOBLING, 1994). Qualquer estímulo sobre esses músculos resultará em impactos significativos no seu crescimento (HOULIHAN \& LAURENT, 1987; JOHNSTON, 2006). Nos peixes estocados na BD e DI, a taxa de crescimento específica (TCE) foi significativamente maior nos grupos exercitados do que nos demais grupos. Porém, foi observada redução nos valores desse índice em função do aumento da densidade. No entanto, a TCE pode variar em razão do peso do peixe, da espécie, do estado nutricional e do manejo, dentre outros fatores (JOBLING, 1994). Assim, BRANDÃO et al. (2005), iniciando a recria de matrinxã com $4,11 \mathrm{~cm}$ e $0,56 \mathrm{~g}$ durante 30 ou 60 dias, em tanques rede com 200, 300, 400 e 500peixes $\mathrm{m}^{-3}$, obtiveram TCE de $10,6 \%$ na primeira fase e $5,2 \%$ na segunda, porém, sem diferenças significativas entre as densidades de estocagem. No presente estudo, iniciou o experimento com peixes maiores $(12,3 \mathrm{~cm}$ e $18,4 \mathrm{~g})$ e, como esperado, a TCE foi inferior. Os valores encontrados variaram significativamente entre 2,8 e $2,4 \%$ no grupo de peixes com exercício, enquanto que, no grupo de peixes sem exercício, esses valores oscilaram entre 2,4 a 1,8\%. Ficou evidente que o exercício moderado melhora a TCE, e o uso de tanques circulares com correnteza moderada otimiza o ambiente e favorece o bom desempenho dos peixes.

A taxa de sobrevivência foi de $100 \%$ no grupo de peixes que nadaram ativamente em todas as densidades, ao passo que, no grupo não exercitado, foi ao redor de 70\% (Tabela 1). Densidades inadequadas podem promover canibalismo em matrinxã (MARQUES et al. 2004). O matrinxã é uma espécie muito agressiva e, quando criada em baixa densidade, é freqüente observar-se alta mortalidade por agressão. Esse comportamento antagônico foi mais pronunciado à noite no grupo sem exercício, visto que no dia seguinte era encontrado um grande número de escamas no filtro mecânico do sistema de recirculação, além de serem observados, na hora da limpeza das caixas, restos de peixes, indicando canibalismo. Isso sugere estabelecimento territorial e aparecimento hierárquico entre os peixes desse grupo. Por outro lado, o grupo de peixes forçados a nadar contra a correnteza não mostrou sinais visíveis de agressões físicas. Segundo JOBLING et al. (1993), o exercício moderado muda o comportamento de salmonídeos, fazendo com que eles se orientem contra a correnteza e comecem a nadar em cardume, diminuindo com isso as agressões antagonistas e a mortalidade. Na recria de matrinxã em 
Tabela 1 - Efeito da natação sustentada e densidade de estocagem sobre o desempenho e a composição proximal de músculo branco e músculo vermelho em juvenis de Brycon amazonicus.

\begin{tabular}{|c|c|c|c|c|c|c|}
\hline \multirow[t]{2}{*}{ Parâmetro } & \multicolumn{3}{|c|}{------Exercício de natação $\left(1,0 \mathrm{cc} \mathrm{s}^{-1}\right)$------ } & \multicolumn{3}{|c|}{---------Controle (água parada)--------- } \\
\hline & $\mathrm{BD}$ & DI & $\mathrm{AD}$ & $\mathrm{BD}$ & DI & $\mathrm{AD}$ \\
\hline PI (g) & $18,2 \pm 3,2$ & $19,3 \pm 2,1$ & $18,4 \pm 2,2$ & $17,0 \pm 2,7$ & $18,8 \pm 1,70$ & $20,7 \pm 2,2$ \\
\hline $\mathrm{PF}(\mathrm{g})$ & $128,3 \pm 15,2^{\mathrm{AB}}$ & $136,5 \pm 13,1^{\mathrm{A}}$ & $103,7 \pm 16,2^{\mathrm{C}}$ & $106,8 \pm 20,7^{\mathrm{BC}}$ & $90,8 \pm 18,2^{\mathrm{CD}}$ & $73,6 \pm 20,3^{\mathrm{D}}$ \\
\hline $\mathrm{CF}$ & $20,4 \pm 1,8^{\mathrm{AB}}$ & $20,0 \pm 1,5^{\mathrm{A}}$ & $19,6 \pm 1,3^{\mathrm{C}}$ & $19,8 \pm 1,5^{\mathrm{BC}}$ & $18,8 \pm 1,2^{\mathrm{CD}}$ & $17,0 \pm 1,6^{\mathrm{D}}$ \\
\hline SV & $100^{\mathrm{A}}$ & $100^{\mathrm{A}}$ & $100^{\mathrm{A}}$ & $67,4 \pm 1,8^{\mathrm{C}}$ & $66,2 \pm 1,6^{\mathrm{C}}$ & $76,8 \pm 2,1^{\mathrm{B}}$ \\
\hline $\mathrm{UP}(\%)^{2}$ & $50,2 \pm 1,3^{\mathrm{C}}$ & $80,4 \pm 1,5^{\mathrm{A}}$ & $71,8 \pm 2,3^{\mathrm{B}}$ & $39,5 \pm 2,2^{\mathrm{D}}$ & $68,5 \pm 4,3^{\mathrm{B}}$ & $65,7 \pm 2,5^{\mathrm{B}}$ \\
\hline FC & $1,012^{\mathrm{B}}$ & $1,080^{\mathrm{A}}$ & $1,013^{\mathrm{B}}$ & $0,964^{\mathrm{B}}$ & $0,965^{\mathrm{B}}$ & $0,981^{\mathrm{B}}$ \\
\hline CAA & $1,3 \pm 0,1^{\mathrm{A}}$ & $1,3 \pm 0,1^{\mathrm{A}}$ & $1,2 \pm 0,1^{\mathrm{A}}$ & $1,7 \pm 0,1^{\mathrm{B}}$ & $1,7 \pm 0,2^{\mathrm{B}}$ & $1,9 \pm 0,1^{\mathrm{C}}$ \\
\hline $\mathrm{TCE}^{3}$ & $2,8 \pm 0,1^{\mathrm{AB}}$ & $2,9 \pm 0,1^{\mathrm{A}}$ & $2,4 \pm 0,1^{\mathrm{B}}$ & $2,4 \pm 0,1^{\mathrm{B}}$ & $2,4 \pm 0,1^{\mathrm{BC}}$ & $1,8 \pm 0,1^{\mathrm{C}}$ \\
\hline \multicolumn{7}{|c|}{ Composição corporal de MB } \\
\hline Prt & $20,2 \pm 0,7$ & $20,7 \pm 0,5$ & $20,2 \pm 0,8$ & $19,2 \pm 0,7$ & $19,3 \pm 0,7$ & $19,1 \pm 0,6$ \\
\hline Et & $1,8 \pm 0,4$ & $1,7 \pm 0,3$ & $1,6 \pm 0,7$ & $1,1 \pm 0,2$ & $1,4 \pm 0,4$ & $1,4 \pm 0,4$ \\
\hline Um & $77,9 \pm 0,8$ & $77,7 \pm 0,5$ & $78,2 \pm 0,9$ & $79,2 \pm 0,8$ & $79,1 \pm 0,6$ & $79,5 \pm 0,6$ \\
\hline \multicolumn{7}{|c|}{ Composição corporal de MV } \\
\hline Prt & $18,2 \pm 1,3$ & $18,4 \pm 1,5$ & $18,6 \pm 2,7$ & $18,1 \pm 0,7$ & $17,8 \pm 2,3$ & $17,5 \pm 2,2$ \\
\hline Et & $7,4 \pm 0,9^{\mathrm{B}}$ & $8,1 \pm 1,6^{\mathrm{AB}}$ & $9,6 \pm 1,1^{\mathrm{A}}$ & $7,7 \pm 1,3^{\mathrm{AB}}$ & $6,9 \pm 0,9^{\mathrm{B}}$ & $6,7 \pm 1,2^{\mathrm{B}}$ \\
\hline Um & $73,5 \pm 2,3^{\mathrm{AB}}$ & $73,5 \pm 1,7^{\mathrm{AB}}$ & $71,6 \pm 1,0^{\mathrm{B}}$ & $74,1 \pm 1,2^{\mathrm{AB}}$ & $75,2 \pm 1,1^{\mathrm{A}}$ & $75,8 \pm 1,8^{\mathrm{A}}$ \\
\hline
\end{tabular}

$\mathrm{BD}=$ Baixa densidade de estocagem $\left(88\right.$ peixes $\left./ \mathrm{m}^{3}\right) ; \mathrm{DI}=$ Densidade de estocagem intermediária $\left(176\right.$ peixes $\left./ \mathrm{m}^{3}\right) ; \mathrm{AD}=\mathrm{Alta}$ densidade de estocagem (353peixes $/ \mathrm{m}^{3}$ ); PI=peso inicial; $\mathrm{PF}=$ peso final; $\mathrm{CF}=$ comprimento final; $\mathrm{SV}=$ sobrevivência; $\mathrm{UP}=$ uniformidade de peso (Peixes que estão $20 \%$ acima ou abaixo do peso médio de cada tratamento); $\mathrm{FC}=$ fator de condição; $\mathrm{CAA}=$ conversão alimentar aparente; TCE=Taxa de crescimento específico; $\mathrm{MB}=$ músculo branco; $\mathrm{MV}=$ músculo vermelho; Prt=proteína; Et=Extrato etéreo; Um=Umidade. Os valores de Prt, Et e Um da composição corporal estão expressos em (\%), em base úmida. Médias seguidas por letras diferentes na mesma linha são significativamente diferentes $(\mathrm{P}<0,05)$. Os valores estão expressos em média $\pm \mathrm{DP}$.

tanque rede, altas taxa de sobrevivência dependem da qualidade da água, do tamanho e da condição do alevino, e do manejo alimentar, dentre outros fatores (BRANDÃO et al., 2005). Entretanto, a sobrevivência dos peixes também pode ser aumentada por mudanças comportamentais ocasionadas pelo exercício moderado, como verificado neste estudo.

A conversão alimentar (CA) sofreu influência da atividade física. Peixes treinados a nadar de forma moderada e criados nas diferentes densidades mostraram melhor conversão alimentar $(\mathrm{P}<0,05)$ que aqueles criados em sistemas convencionais de água parada, como pode ser verificado pelos baixos valores da CA (Tabela 1 ), embora tenham consumido maior quantidade de alimento $\left(1,60 \mathrm{~g}\right.$ peixe $\left.\mathrm{dia}^{-1}\right)$ que o controle $(1,40 \mathrm{~g}$ peixe $\left.{ }^{-1} \mathrm{dia}^{-1}\right)$. Mesmo assim, esses peixes conseguiram ganhar maior peso por unidade de alimento consumido. Estudos similares com bagre do canal (Ictalurus punctatus) também mostraram melhor CA em espécimes estimulados a nadar ativamente, em comparação com os animais criados em repouso (JARBOE \& GRANT, 1996). Respostas similares também foram observadas em salmonídeos (JOBLING, 1994; DAVISON, 1997) e em matrinxã (HACKBARTH \& MORAES, 2006). Sob condições de exercício, a agressividade diminui e, com isso, mais energia é disponibilizada para o crescimento somático (JOBLING et al. 1993), melhorando dessa forma, a eficiência alimentar.

A composição corporal de peixes criados em cativeiro pode ser manipulada primariamente pela qualidade e quantidade de nutrientes da dieta, pelo nível de arraçoamento e pelo regime alimentar, dentre outros fatores (SHEARER, 1994; JOBLING, 2001). Outros fatores (como o exercício) podem ocasionar mudanças marcantes na concentração dos constituintes corporais (DAVISON, 1997). Neste estudo, a composição corporal dos músculos natatórios dos juvenis de matrinxã apresentou mudanças significativas em resposta às condições impostas tanto pelo exercício induzido, quanto pela densidade de estocagem (Tabela 1). O teor médio de proteína do músculo branco do grupo de peixes submetido ao exercício de natação sustentada foi 5,8\% maior $(\mathrm{P}<0,05)$ que o do grupo de peixes criado em água parada. $\mathrm{O}$ conteúdo de água desse músculo foi $2 \%$ menor $(\mathrm{P}<0,05)$ que o de peixes sem exercício. Similarmente, o músculo vermelho dos peixes submetidos ao exercício mostrou um aumento de 
lipídios e uma diminuição de umidade em função do aumento da densidade de estocagem. Entretanto, o músculo vermelho não apresentou mudanças significativas na sua composição nos peixes não exercitados para as diferentes densidades (Tabela 1). Isso ao ultrapassar pode indicar o custo adaptativo ao adensamento, isto é, o limiar de estocagem da espécie, a demanda energética é aumentada para manutenção da homeostese (VIJAYAN, M.M. et al. 1990). Em relação à condição física, foi observado que a deposição de lipídios no músculo vermelho do grupo de peixes sob exercício nas diferentes densidades foi em média $15 \%$ maior $(\mathrm{P}<0,05)$ do que no grupo de peixes considerado sedentário. Por sua vez, o conteúdo de umidade no músculo vermelho apresentou um perfil oposto aos lipídios. Os peixes exercitados exibiram nesse tipo de músculo $3 \%$ menos de umidade $(\mathrm{P}<0,05)$ que aqueles mantidos em condições sedentárias (Tabela 1).

Os resultados deste estudo mostraram que os músculos natatórios do matrinxã apresentaram grande plasticidade ao exercício, mudando a sua composição conforme as exigências. Os peixes exercitados depositaram mais proteína em seus músculos. O maior crescimento nos peixes se deve principalmente à hipertrofia das fibras musculares (TOTLAND et al., 1987; JOHNSTON, 2006). Dependendo da duração e intensidade do exercício, a composição corporal dos peixes muda (DAVISON \& GOLDSPINK, 1977). Houve aumento de lipídios tanto no músculo vermelho, quanto no músculo branco da truta marrom Salmo trutta, em velocidade de natação de $1,5 \mathrm{cc} \mathrm{s}^{-1}$. Em velocidades maiores $\left(3,0 \mathrm{cc} \mathrm{s}^{-1}\right)$, os lipídios de ambos os tipos de músculo diminuíram, indicando que foram usados como fonte calórica. Por outro lado, CHRISTIANSEN et al., (1989) relatam aumento de proteína e diminuição de lipídios na composição corporal do Salvelinus alpinus, exercitados entre 2,3 a $1,2 \mathrm{cc} \mathrm{s}^{-1}$.

$\mathrm{O}$ conceito de que peixes criados sob correnteza constante teriam menor crescimento, menor concentração de lipídios e maior conteúdo de água no corpo, quando comparados a peixes criados em sistemas convencionais de água parada, deve-se à hipótese de que altos níveis de energia seriam requeridos para manter sua posição contra a corrente de água (HERNÁNDEZ et al., 2002). Entretanto, os resultados obtidos com juvenis de matrinxã mostram o contrário, uma vez que, quando criados em velocidades moderadas, apresentam maiores taxas de crescimento refletidas em maior deposição de proteína e lipídios corporais. É importante ressaltar que o aumento na deposição de gordura nos músculos natatórios do matrinxã, como resposta adaptativa ao exercício de natação sustentável, pode conferir maior sabor à carne. Em condições normais de criação em água sem movimento e geralmente quando há excesso de energia na dieta, o acúmulo de gordura é visceral, a qual é indesejada pela indústria do processamento por diminuir o rendimento da carcaça. Provavelmente, a gordura que se acumularia nas vísceras do grupo de peixes exercitados foi, em parte, utilizada como combustível para a locomoção, sendo outra mobilizada e depositada, principalmente, no músculo vermelho, como reserva energética. Embora, esse músculo possa armazenar cerca de quatro vezes mais lipídios que o músculo branco, sua função primordial é metabolizálos in situ, especialmente durante a natação sustentada. Por sua vez, a gordura visceral está mais relacionada ao local de armazenamento (JOBLING, 2001). Os depósitos viscerais de lipídios são facilmente mobilizados e declinam quase imediatamente em situações de demanda contínua de energia, tais como restrição alimentar e migração reprodutiva (VAN DEN THILLART \& VAN RAAIJ, 1995). Segundo esses autores, lipídios viscerais de espécies como truta, carpa e enguia diminuem significativamente diante da alta demanda metabólica, enquanto os teores de lipídios hepáticos e musculares não são afetados. Portanto, a depleção dos lipídios desses tecidos é mais lenta que a dos lipídios viscerais.

Em conclusão, a criação de juvenis de matrinxã sob condições de exercício moderado e em densidade intermediaria (176 peixes $\mathrm{m}^{-3}$ ) é a mais indicada por apresentar melhor desempenho. O exercício de natação moderado independente da densidade de estocagem traz mudanças na composição corporal de juvenis de matrinxã que se refletem principalmente em maior deposição de proteína no músculo branco e de lipídios no músculo vermelho. Isso possibilita aumento de reservas energéticas e maior capacidade de resistência, além de melhor adaptação às condições de adensamento em sistemas intensivos de criação.

\section{REFERÊNCIAS}

ARANÄRÄ A.; BRÄNNÄS, E. Dominance-feeding behaviour in Arctic charr and rainbow trout: the effect of stocking density. Journal of Fish Biology, v.48, p.242-254, 1996.

AOAC. Official methods of analyses of the Association of Agriculture Chemists. 15.ed. Washington DC, 1990. 1008 p.

ARBELÁEZ-ROJAS , G.A. Efeitos da natação sustentada no crescimento, na densidade de estocagem e na composição corporal em juvenis de matrinxã, Brycon amazonicus. Aspectos adaptativos e respostas metabólicas. 
2007. 149f. Tese (Doutorado em Ecologia e Recursos Naturais) - Universidade Federal de São Carlos. São Carlos, SP, Brasil

BARTON, B.A. Stress in fishes: a diversity of responses with particular reference to changes in circulating corticosteroids. Integrative and Comparative Biology, v.42, p.517-525 2002

BRANDÃO, F.R. et al. Densidade de estocagem de matrinxã, (Brycon amazonicus) na recria em tanque-rede. Pesquisa Agropecuária Brasileira, v.40, p.299-303, 2005.

BOYD, C.E. Water quality in ponds for aquaculture. Alabama: Auburn University, 1990. 482p.

CHRISTIANSEN, J.S. et al. Effects of Sustained Exercise on Growth and Body Composition of First-Feeding Fry of Arctic charr, Salvelinus alpinus (L.). Aquaculture, v.79, p.329$335,1989$.

CHRISTIANSEN, J.S. et al. The combined effects of stocking density and exercise on the behaviour, food intake, and growth of juvenile Arctic charr (Salvelinus alpinus L.). Canadian Journal of Zoology, v.70, p.115-122, 1992.

DAVISON, W.; GOLDSPING, G. The effect of prolonged exercise on the lateral musculature of the Brown trout Salmo truta. Journal Experimental Biology, v.70, p.1-12, 1977.

DAVISON, W. The effects of exercise training on teleost fish, a review of recent literature. Comparative Biochemistry Physiology, v.117A, p.67-75, 1997.

ELLIS, T. et al. The relationships between stocking density and welfare in farmed rainbow trout. Journal of Fish Biology, v.61, p.493-531, 2002.

FURUYA, W.M. et al. Dietas peletizada e extrusada para machos revertidos de tilápia do Nilo, (Oreochromis niloticus) em fase de terminação. Ciência Rural, v.28, n.3, p.483-487, 1998.

GOMES, L.C. et al. Effect of stocking on quality, survival, and growth of larvae of matrinxã, Brycon cephalus (Characidae), in ponds. Aquaculture, v.183, p.73-81, 2000

HACKBARTH, A.; MORAES, G. Biochemical responses of matrinxã, Brycon cephalus (Gunther, 1869) after sustained swimming. Aquaculture Research, v.37, p.1070-1078, 2006 .

HERNÁNDEZ, M.D. et al. Effects of intensive exercise training on rainbow trout growth, body composition and metabolic responses. Journal Physiology and Biochemistry, v.58, p.1-8, 2002.

HOULIHAN, D.F.; LAURENT, P. Effect of exercise training on the performance, growth, and protein turnover of rainbow trout (Salmo gairdneri). Canadian Journal Fisheries and Aquatic Sciences, v.44, p.1614-1621, 1987.

HOWES, G.J. Review of the genus Brycon (Teleostei: Characoidei). Bulletin of the British Museum Natural History and Zoology, v.43, p.1-47, 1982.
INOUE, L.A.K.A. et al. Clove oil as anesthetic for juveniles of matrinxã, Brycon cephalus (Gunther, 1869). Ciência Rural, v.33, p.943-947, 2003.

JARBOE, H.H.; GRANT, W.J. The effects of water velocity on the growth, dressout, and body composition of channel catfish, Ictalurus puntactus, raised in circular tanks. Journal of Applied Aquaculture, v.6, n.3, p.13-21, 1996.

JOBLING, M. Fish bioenergetics. London: Chapman \& Hall, 1994. 309p.

JOBLING, M. et al. The effects of prolonged exercise training on growth performance and production parameters in fish. Aquaculture International, v.1, p.95-111, 1993.

JOBLING, M. Nutrient partitioning and the influence of feed composition on body composition. In. HOULIHAN, D. et al. Food intake in fish. Oxford-UK: Blackwell-science, 2001. Chap.15, p.354-375.

JOHNSTON, I.A. Environment and plasticity of myogenesis in teleost fish. Journal of Experimental Biology, v.209, p.2249-2264, 2006

KESTEMONT, P.; BARAS, E. Environmental factors and feed intake: mechanisms and interactions. In. HOULIHAN, D. et al. Food intake in fish. Oxford-UK: Blackwell-science, 2001. Chap.6, p.131-145.

MARQUES, N.R. et al. Influência da densidade de estocagem no cultivo de alevinos de matrinxã Brycon cephalus (Günther, 1869) em condições experimentais. Acta Scientiarum. Biological Sciences, v.26, p.55-59, 2004.

PAPST, M.H. et al. Effect of rearing density on the early growth and variation in growth of juvenile Arctic Salvelinus alpinus. Aquaculture and Fisheries Management, v.23, 41-47, 1992

ROCHA R.M. et al. Physiological responses associated with capture and stress in matrinxã Brycon cephalus (Gunther, 1869). Aquaculture Research, v.35, p.245-249, 2004.

ROCHA, Y.R. et al. Aspectos nutritivos de alguns peixes da Amazônia. Acta Amazônica, v.12, n.4, p.787-794, 1982.

SHEARER, K.D. Factors affecting the proximal composition of cultured fishes with emphasis on salmonids. Aquaculture, v.119, p.63-88, 1994

SPIX, J.B VON; AGASSIZ, L. Selecta genera et species piscium quos in itinere per Brasiliam annos MDCCCXVII-MDCCXX jussu et auspiciis Maximiliani Josephil. Colleget et pingendso curavit J.B. de Spix. Monachii. Selectarum Brasilian, Part 1: $\mathrm{i}-\mathrm{xvi}+\mathrm{i}-\mathrm{ii}+1-82$, pis, p.1-48, 1829.

TOTLAND, G.K. et al. Growth and composition of the swimming muscle of adult Atlantic salmon, Salmo salar during 
long-term sustained swimming. Aquaculture, v.66, p.299$313,1987$.

VAN DEN THILLART, G.; VAN RAAIJ, M. Circulatory substrate fluxes and their regulation. In: HOCHACHKA, P.W., MOMMSEN, T.P. (Eds.). Metabolic biochemistry, biochemistry and molecular biology of fish. Amsterdam: Elsevier, 1995. V.4, p.14-63.

VIJAYAN, M.M. et al. High stocking density alters the energy metabolism of brook charr, Salvelinus fontinalis. Aquaculture, v.88, p.371-381, 1990 .
VINATEA, A.L. Princípios químicos da qualidade da água em aqüicultura. Florianópolis: EDUFSC, 2004. 231p.

WEDEMEYER, G.A. Effects of rearing conditions on the health and physiological quality of fish in intensive culture. In: IWAMA, K. et al. Fish stress and health in aquaculture. Cambridge: Cambridge University, 1997. p.35-71.

ZANIBONI FILHO, E. et al. Potencial del género Brycon en la piscicultura Brasileña. Rev Colcienc Pec, v.19, n.2, p.233$240,2006$. 